\title{
The Variable Polarization XUV Beamline P04 at PETRA III: Optics, Mechanics and their Performance
}

\author{
Jens Viefhaus ${ }^{\mathrm{a}, *}$, Frank Scholz ${ }^{\mathrm{a}}$, Sascha Deinert ${ }^{\mathrm{a}}$, Leif Glaser ${ }^{\mathrm{a}}$, \\ Markus Ilchen ${ }^{\mathrm{a}}$, Jörn Seltmann ${ }^{\mathrm{a}}$, Peter Walter ${ }^{\mathrm{a}}$, Frank Siewert ${ }^{\mathrm{b}, * *}$ \\ ${ }^{a}$ Deutsches Elektronen-Synchrotron DESY, Notkestraße 85, 22607 Hamburg, Germany \\ ${ }^{b}$ Helmholtz-Zentrum Berlin (HZB) für Materialien und Energie, \\ Albert-Einstein-Straße 15, 12489 Berlin, Germany
}

\begin{abstract}
The layout of the Variable Polarization XUV Beamline P04 at PETRA III is described with emphasis on selected examples of optics, mirrors and gratings. A precise characterization of the optics, their performance inside the holder and of the surrounding mechanics is presented. This also includes a detailed characterization of the different beamline mechanics as a whole (grating unit, exit slit unit, re-focusing unit) including the environment.
\end{abstract}

Keywords: synchrotron radiation, x-ray optics, optic metrology, VLS-PGM

\section{Introduction}

The $6 \mathrm{GeV}$ storage ring PETRA III [1] started operation in 2009 as one of the most powerful x-ray synchrotron radiation sources in the world. One of its 14 operating beamlines, the Variable Polarization XUV Beamline P04, provides radiation in the photon energy range from 250 to $3000 \mathrm{eV}$. Due to the low emittance of the storage ring of $1 \mathrm{nmrad}$ and the $5 \mathrm{~m}$ APPLE-II-type undulator installed at beamline P04, the radiation is of very high quality in terms of brightness and coherence. In order to exploit the opportunities offered by these radiation properties, a selection of scientific fields and tech-

\footnotetext{
*Principal corresponding author

** Corresponding author

Email addresses: jens.viefhaus@desy.de (Jens Viefhaus), frank.siewert@helmholtz-berlin.de (Frank Siewert)
} 
niques has been identified in the planning stage of the PETRA III project. The selection contains:

- Gas phase studies

- Soft x-ray scattering

- Magnetic spectroscopy

- High-resolution photoelectron spectroscopy

- Surface chemistry

- Spectro-microscopy

In order to achieve the associated scientific goals, certain beamline specifications have been defined during a number of user workshops. The required specifications are:

- Photon energy range: $(<100) 250-3000 \mathrm{eV}$

- Resolving power: $>10^{4}$ (up to $>3 \times 10^{4} @ 1 \mathrm{keV}$ )

- Photon flux: $>10^{12}$ photons/s (up to $5 \times 10^{12}$ photons $/ \mathrm{s}$ )

- Spot size at sample: $10 \times 10 \mu m^{2} / 50 \times 50 \mu m^{2}$

- Polarization: circular, linear (hor./vert./rot.) ( $<0.1 \mathrm{~Hz}$ switching rate)

\section{Beamline design}

The combination of all these parameters is unique for a XUV beamline, therefore we had to adopt the optical design given in figure 1 especially to be able to cover the required large range of photon energies. As there is no standard coating material available which has no absorption edge in this photon energy range, we had to use at least two coatings on the optical elements. This in turn favored the use of plane optical elements in order to use a simple translation for a change of the coating without the need for re-alignment. As the photon beam divergence and also the vertical source size is already diffraction limited in the full energy range, one may use the un-collimated beam up to the focusing optics in front of the experiment. 


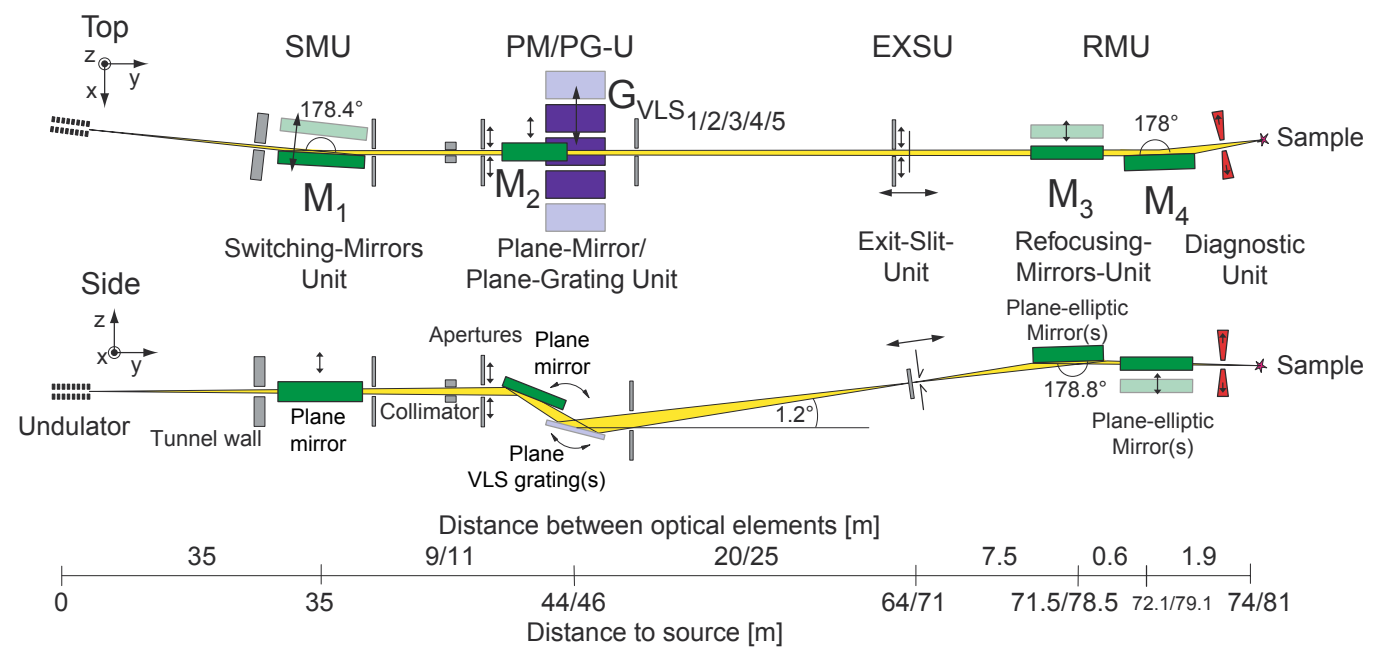

Figure 1: Schematic layout of the Variable Polarization XUV Beamline P04 at PETRA III. The main opto-mechanical components are labeled. This includes the switching mirrors unit (SMU) which distributes the radiation in one of the two branch lines. As a consequence of this, all following components need to be realized twice: the plane-mirror/planegrating-units (PM/PG-U), the exit slit units (EXSU) as well as the refocusing mirrors units (RMU). Both RMU can operate two sets of fixed KB-focusing mirror pairs each having two different foci. All optical elements have to be translatable in order to access the two different coatings $(\mathrm{Au} / \mathrm{Pt}$ or $\mathrm{Rh}$, respectively) required to cover the full range of photon energies. 
Therefore the first two optical elements ( $\mathrm{M}_{1}$ and $\mathrm{M}_{2}$, respectively) can be plane mirrors.

Mirror $\mathrm{M}_{1}$ is situated inside the switching mirrors unit (SMU) which allows to serve two branch lines of the beamline P04 enabling a fast switching between two independent experiments. Each branch may be adapted to the specific needs of different experiments. Mirror $\mathrm{M}_{1}$ has to withstand a rather high power of up to $1.5 \mathrm{~kW}$ (at $100 \mathrm{~mA}$ storage ring current). Power(density)-[2,3] and FEM-calculations showed, that only internal $\mathrm{lN}_{2}$ cooling keeps the deformations due to the heat load within acceptable limits. In contrast to this, FEM-analysis indicated that internal water cooling is sufficient for mirror $\mathrm{M}_{2}$ which is the pre-mirror in front of the grating and is expected to absorb more than one order of magnitude less power (up to $48 \mathrm{~W}$ at $100 \mathrm{~mA}$ ). The focusing onto the exit slit is performed by a varied line-space (VLS) plane grating. Due to the long exit arm length only a modest variation of the line density is required. The focusing at the exit slit remains at a constant position if the so-called fixed focus constant $c_{f f}$ is varied accordingly with varying photon energy [4]. A further modification of the $c_{f f}$ parameter could allow to compensate changes of the optics due to thermal deformation [5]. In any case this constant is fully determined for a particular grating and different modes of operation e.g. normal vs. high resolution can only be obtained with different gratings. To allow for different modes of operation, up to five gratings $\left(\mathrm{G}_{1-5}\right)$ can be installed in the plane-mirror/plane grating-unit (PM/PG-U). The focusing onto the different sample areas is performed via two different pairs of mirrors $\left(\mathrm{M}_{3}\right.$ and $\mathrm{M}_{4}$ ) having a fixed shape in Kirkpatrick-Baez(KB)-geometry [6] in order to achieve simultaneously a vertical and horizontal focus at the given sample plane. The individual adjustment of the horizontal focusing mirror allows to reduce an unwanted effect due to the thermal deformation of mirror $\mathrm{M}_{1}$ namely the change of the effective source distance and hence of the effective demagnification ratio of the optics.

\section{Mechanics}

The anticipated beamline performance imposes also very stringent stability requirements on the mechanics of the optics as well as the overall stability of the facility. The latter one is based on a $1 \mathrm{~m}$ thick concrete foundation covering the entire floor of the experimental hall, sized $280 \mathrm{~m}$ in length and $24 \mathrm{~m}$ in width [7]. This way both the storage ring section including the undu- 
lators as well as all beamlines are based on the same single piece of concrete resulting in a damping of the vibrations due to environmental noise by one order of magnitude [8]. Despite its large volume $\left(80000 \mathrm{~m}^{3}\right)$ the experimental hall at PETRA III is temperature controlled to a stability within $1^{\circ} \mathrm{C}$ througout the whole year.

The mechanics which are placed inside ultra-high-vacuum vessels have been designed for high accuracy and stability of the required movements. All mechanics have been thoroughly tested prior, during and after the installation. In all cases the required specifications have been met. As an example the mechanical performance of the plane-mirror/plane-grating unit $(\mathrm{PM} / \mathrm{PG}-\mathrm{U})$ is shown in figure 2 for the case of the rotational movement of the plane grating. The measurement is performed simultaneously by the internal in-vacuum angular measuring system (Heidenhain RON 905 UHV, interpolated resolution 0.002 arcsec) which is also used for the closed-loop control, as well as an external measurement using an autocollimator outside the vacuum (Elcomat HR, noise floor 0.004 arcsec, 0.01 arcsec specified accuracy). The results in figure 2 show clearly resolved steps of 0.02 arcsec which are necessary in order to utilize the specified resolution of the beamline P04. Because of the fact that the external device is fixed to the base of the PM/PG-U with an aluminum frame support, certain influences from external vibrations can not be completely ruled out. Therefore, more precise measurements with sub-0.01-arcsec steps - which are at the limits of the present setup in ideal situations during the night at weekends - would have to use a different setup, e.g. using in-vacuum interferometers. Nevertheless, the overall results of all mechanics tests make us extremely confident about the future performance. For the exit-slit unit (EXSU), having a vertical exit slit opening of $0-2000 \mu \mathrm{m}$, we verified a repeatability of the slit setting in the order of $100 \mathrm{~nm}$ in the range up to $100 \mu \mathrm{m}$ and less than $1 \%$ deviation above. The refocusing mirrors units based on two out-of-vacuum hexapods for each of the KB-mirrors showed a stability of the mirror movement better than $1 \mu \mathrm{m}$ and an angular precision in the order of $1 \mu \mathrm{rad}$ which can be further improved down to about $0.1 \mu \mathrm{rad}$ for the case of the two pitch angles of the mirrors $\mathrm{M}_{3}$ and $\mathrm{M}_{4}$ using in-vacuum piezo-actuators. In all cases it turned out to be necessary that all of the mechanics are installed inside a separate climatization hutch which is controlled to a temperature stability within $0.1^{\circ} \mathrm{C}$ which is an order of magnitude better than the experimental hall at PETRA III. 


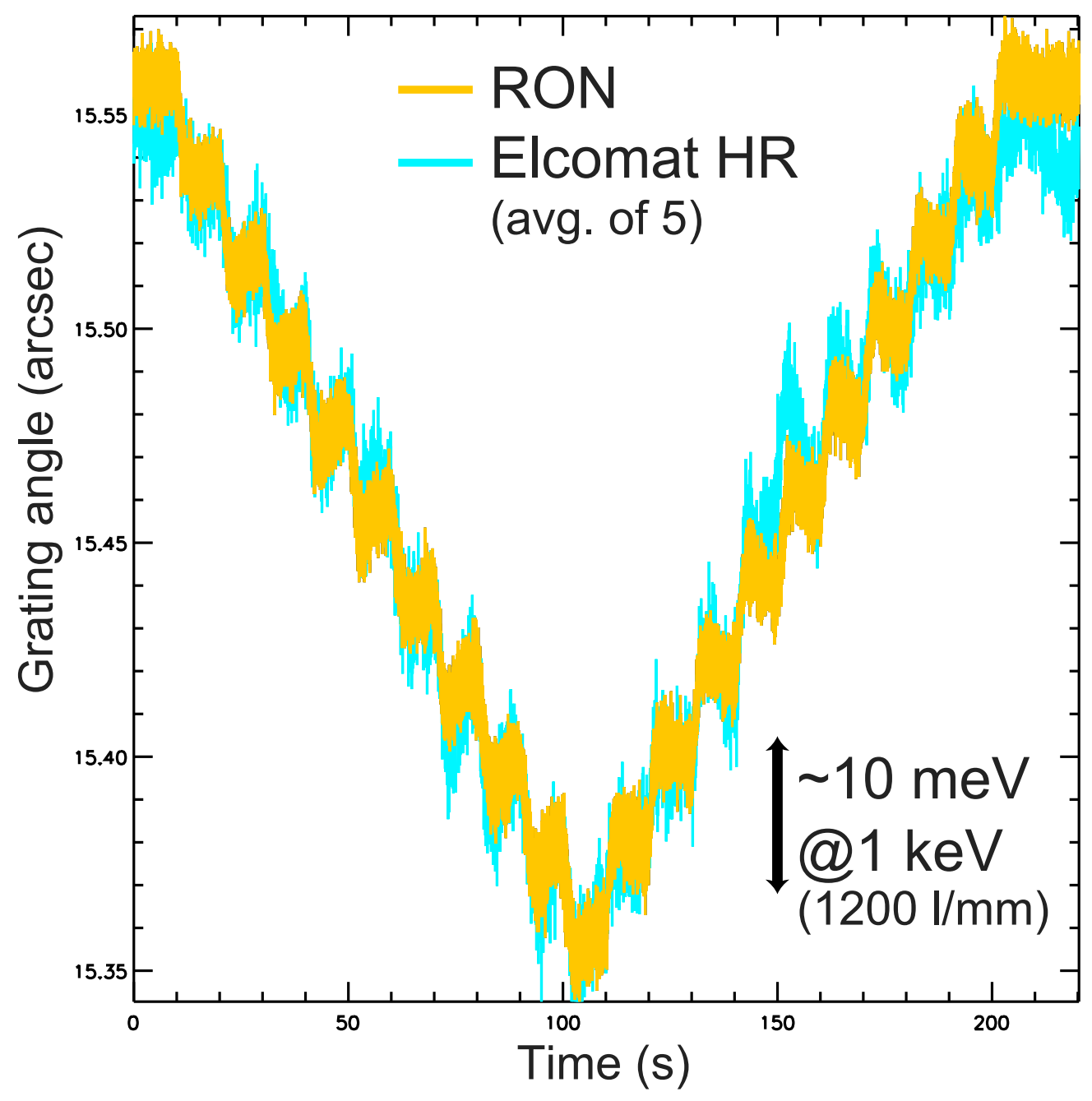

Figure 2: Mechanical performance of the rotational movement of the grating inside the PM/PG-U vessel of beamline P04 (branch 2). The curves showing the internally (RON) as well as externally (Elcomat HR) measured rotation are obtained by a stepwise rotation of the grating. Each step corresponds to $0.02 \mathrm{arcsec}$ with an interval of $10 \mathrm{~s}$ between each of the consecutive steps. An arrow indicates the corresponding rotation of the $1200 \mathrm{l} / \mathrm{mm}$ grating for a $10 \mathrm{meV}$ energy step at $1 \mathrm{keV}$ photon energy. 


\section{Optics}

In order to achieve the specified beamline performance, correspondingly high quality optics at the limit of present technology have to be obtained. This is both a challenge for the manufacturer as well as for quality control. All optics have been characterized at the BESSY II Optics Laboratory of the Helmholtz Zentrum Berlin using the nanometer optical component measuring machine (NOM) for shape measurements [9] as well as a white light interferometer (magnification $20 \times$ and $50 \times$ ) and an AFM for roughness determination. A collection of the results obtained so far together with the required specifications is presented in table 1 . The results show that in all cases the specifications have been met. For the case of the grating we also verified that the slope error of the plane substrate does not change if mounted properly in the grating holder.

Table 1: Properties of the P04 optics

\begin{tabular}{|l||l|l|c|c|c|c|}
\hline \hline Item & Size & Figure & \multicolumn{2}{|c|}{ Roughness $(\mathrm{rms}$ in nm $)$} & \multicolumn{2}{|c|}{ Slope error (rms in arcsec) } \\
\hline & $(1 \times \mathrm{w} \times \mathrm{h}, \mathrm{mm})$ & & Specified & Measured & Specified & Measured \\
\hline \hline $\mathrm{M}_{1}$ & $400 \times 60 \times 60$ & plane & 0.5 & & 0.1 & 0.06 \\
$\mathrm{M}_{2}$ & $560 \times 60 \times 60$ & plane & 0.5 & & 0.05 & \\
$\mathrm{G}_{1}$ & $145 \times 32 \times 23$ & plane, $400 \mathrm{l} / \mathrm{mm}$ & 0.5 & $0.3-0.4$ & 0.05 & 0.037 \\
$\mathrm{G}_{2}$ & $130 \times 32 \times 23$ & plane, $1200 \mathrm{l} / \mathrm{mm}$ & 0.5 & $0.5-0.7$ & 0.05 & 0.024 \\
$\mathrm{M}_{3 b 1}$ & $500 \times 30 \times 60$ & plane-ellipse & 0.5 & $<0.1$ & 0.2 & 0.2 \\
$\mathrm{M}_{4 b 1}$ & $600 \times 30 \times 60$ & plane-ellipse & 0.5 & & 0.2 & \\
$\mathrm{M}_{3 b 2}$ & $500 \times 30 \times 60$ & cylinder & 0.5 & 0.1 & 0.2 & 0.09 \\
$\mathrm{M}_{4 b 2}$ & $600 \times 30 \times 60$ & cylinder & 0.5 & 0.1 & 0.2 & 0.13 \\
\hline
\end{tabular}

For a detailed assessment of the general performance of the optics, however, it is necessary to check this by repeating the ray-tracing calculations which had been used to derive the required optical properties a-priori. The calculations took advantage of the software package SHADOW/XOP [10] together with additional procedures developed to automatize repetitive tasks in order to calculate many different photon energies and taking into account misalignments. Using the surface topology derived from the metrology data which has been measured after the manufacturing process we obtained the results shown in figure 3. For those optics where we have not yet obtained the final data we applied the data of comparable components. The results 
are quite encouraging as they show that one is able to match or even exceed the required beamline performance.

Currently beamline P04 is in operation with preliminary optics for the $\mathrm{M}_{1}$ and $\mathrm{M}_{2}$ mirror, therefore we can not give a final answer on the photon energy resolution. As only three of four KB-mirrors have been delivered yet, we can also not give quantitative results on the obtained focus dimensions. However, we can state that the photon flux as well as the overall stability of the beamline and its components is well in accordance with the specifications. As soon as the missing optics will be delivered, characterized and installed we can expect that the intended science program will quickly take advantage of the unique set of photon properties offered by beamline P04 at PETRA III.

It is a pleasure to acknowledge the great support by the PETRA III team and all DESY-FS service groups and workshops. In addition we warmly thank Jana Buchheim (HZB) for valuable assistance during the metrology process as well as the inspiring advice from the P04 review team consisting of Nick Brookes (ESRF), Rolf Follath (HZB), Hermann Franz (DESY), Michael Martins (University of Hamburg), Ruben Reininger (SAS), Fred Senf (HZB), Edgar Weckert (DESY) and Wilfried Wurth (University of Hamburg). We also want to acknowledge the $R \& D$ support of the different vendors of major beamline components namely FMB Berlin (PM/PG-U), Bestec GmbH (ESXU) and FMB Oxford (RMU) as well as the optics vendors InSync, Shimadzu, Pilz Optics and Zeiss.

\section{References}

[1] H. Franz, O. Leupold, R. Röhlsberger, S.V. Roth, O.H. Seeck, J. Spengler, J. Strempfer, M. Tischer, J. Viefhaus, E. Weckert, and T. Wroblewski, Synchrotron Radiation News 19 (2006) 25-29

[2] T. Tanaka and H. Kitamura, Journal of Synchrotron Radiation 8 (2001) 1221-1228

[3] R. Reininger, SRcalc software (2005) v1.4.0

[4] R. Reininger and A.R.B. de Castro, Nuclear Instruments $\&$ Methods in Physics Research Section A-Accelerators Spectrometers Detectors and Associated Equipment 538 (2005) 760-770

[5] R. Reininger, K. Kriesel, S.L. Hulbert, C. Sanchez-Hanke, and D.A. Arena, Review of Scientific Instruments 79 (2008) 033108 

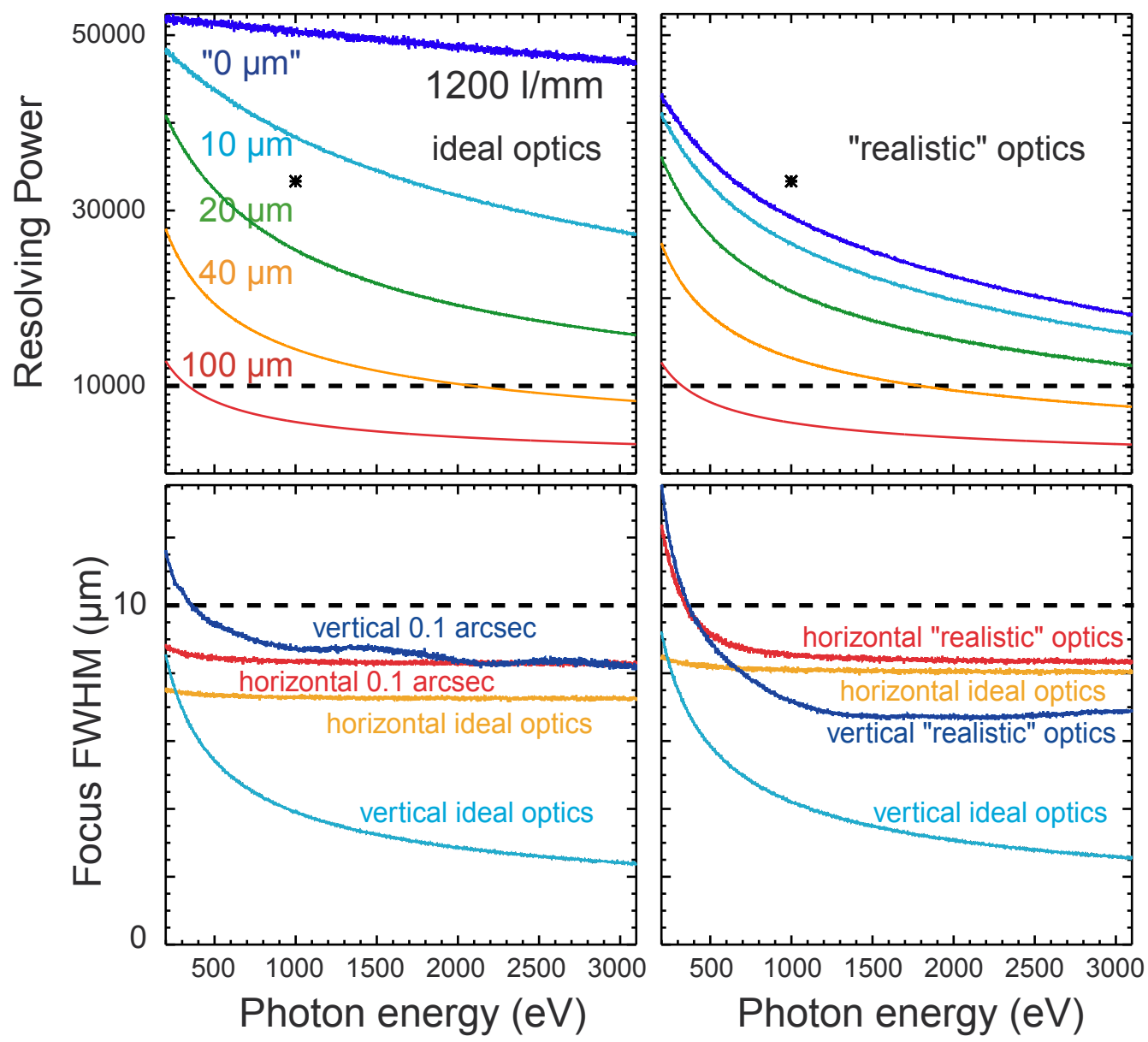

Figure 3: Expected resolving power (top) and focus size at the sample position (bottom) for beamline P04. The results on the left hand side are calculated for perfect optics whereas the results on the right hand side take into account the actually determined shapes of the optics. The dashed line indicates the requested performance by the user groups. The star in the resolving power plots indicates a photon energy resolution of $30 \mathrm{meV}$ at a photon energy of $1 \mathrm{keV}$ which represents a special user demand. 
[6] P. Kirkpatrick and A.V. Baez, Journal of the Optical Society of America 38 (1948) 766-774

[7] H. Franz, L. Hänisch, and J. Spengler, HASYLAB annual report (2006) $145-148$

[8] J. Spengler, H. Franz, and G. Wellenreuther, 11th International Conference on Synchrotron Radiation Instrumentation (2012) TH-G-P-06

[9] F. Siewert, J. Buchheim, S. Boutet, G.J. Williams, P.A. Montanez, J. Krzywinski, and R. Signorato, Optics Express 20 (2012) 4525-4536

[10] M.S. del Rio, N. Canestrari, F. Jiang, and F. Cerrina, Journal of Synchrotron Radiation 18 (2011) 708-716 